\title{
Signaling-based Joint Selection of Wavelengths and Regenerator Points in GMPLS-
} controlled WSONs

Fagertun, Anna Manolova; Cerutti, I.; Andriolli, N.; Sambo, N.; Giorgetti, A.; Castoldi, P.; Ruepp, Sarah Renée

\section{Published in:}

Proceedings of Globecom

Link to article, DOI:

10.1109/GLOCOM.2011.6133743

Publication date:

2011

Link back to DTU Orbit

Citation (APA):

Fagertun, A. M., Cerutti, I., Andriolli, N., Sambo, N., Giorgetti, A., Castoldi, P., \& Ruepp, S. R. (2011). Signalingbased Joint Selection of Wavelengths and Regenerator Points in GMPLS-controlled WSONs. In Proceedings of Globecom IEEE. https://doi.org/10.1109/GLOCOM.2011.6133743

\section{General rights}

Copyright and moral rights for the publications made accessible in the public portal are retained by the authors and/or other copyright owners and it is a condition of accessing publications that users recognise and abide by the legal requirements associated with these rights.

- Users may download and print one copy of any publication from the public portal for the purpose of private study or research.

- You may not further distribute the material or use it for any profit-making activity or commercial gain

- You may freely distribute the URL identifying the publication in the public portal 


\title{
Signaling-based Joint Selection of Wavelengths and Regenerator Points in GMPLS-controlled WSONs
}

\author{
A.V. Manolova ${ }^{1}$, I. Cerutti ${ }^{2}$, N. Andriolli ${ }^{2}$, N. Sambo ${ }^{2}$, A. Giorgetti ${ }^{2}$, P. Castoldi ${ }^{2}$, S. Ruepp $^{1}$ \\ ${ }^{1}$ DTU Fotonik, Kgs. Lyngby, Denmark, e-mail: anva@ @otonik.dtu.dk; ${ }^{2}$ Scuola Superiore Sant’Anna, Pisa, Italy;
}

\begin{abstract}
In wavelength switched optical networks (WSON), quality of transmission (QoT) can be guaranteed by regenerating the optical signal in intermediate nodes. Regeneration can also offer wavelength conversion. When only few regenerators are placed in the WSON, the main issue is to optimally select the nodes where regeneration and wavelength conversion should take place.

This paper proposes a distributed strategy for joint selection of wavelengths and regeneration locations (points) in a dynamic WSON with GMPLS control plane. To support the strategy, extensions to the RSVP-TE protocol are proposed. Simulation results show a significant improvement of the connection blocking and fairness, as well as a reduced amount of used regenerators in the network compared with existing strategies, which are based on disjoint and non-distributed resource selection.
\end{abstract}

\section{INTRODUCTION}

$\mathbf{I}$ $\mathrm{N}$ wavelength switched optical networks (WSON), the quality of transmission (QoT) of the optical signal can be degraded by linear and non-linear physical impairments [1]. Optical-electrical-optical regenerators can recover the signal degradation in specific intermediate nodes of an optical connection by re-amplifying, re-shaping and re-timing the optical signal (referred to as $3 \mathrm{R}$ regeneration). In addition to signal degradation recovery, $3 R$ regenerators offer a native wavelength conversion capability and can be exploited to overcome the wavelength continuity requirements.

Although useful to guarantee QoT and achieve wavelength conversion, $3 \mathrm{R}$ regenerators must be used with parsimony because they are expensive and power hungry devices that limit the optical transparency. Therefore, a number of studies have addressed the issue of optimally placing a limited number of regenerators during the WSON network planning [2]. But it is the control plane, e.g., the Generalized Multi-Protocol Label Switching (GMPLS) control plane [3], that decides in which node(s) regeneration should take place (regeneration point) and where wavelength conversion should be performed (WC point) for each requested connection (i.e., label switched path - LSP). Such decision is taken based on the estimated QoT and on the information about the available wavelengths and $3 \mathrm{R}$ regenerators. Under the GMPLS framework, the reservation of the wavelengths is carried out in a distributed fashion by the resource-reservation protocol with traffic engineering extension (RSVP-TE) [4].

This work has been partially supported by BONE-project ("Building the Future Optical Network in Europe"), a Network of Excellence funded by the European Commission through the 7th ICT-Framework Programme.
Different strategies for RSVP-TE have been proposed for selecting either the regeneration points or the wavelengths. The work in [5] proposed a strategy for RSVP-TE to select wavelengths with the aim of minimizing the wavelength conversion in the network. However, this work does not consider QoT requirements nor 3R selection. The work in [6] proposes distributed strategies to designate the regeneration nodes in GMPLS networks per LSP request. Such strategies are devised to be applied at either the source node, the destination node or the intermediate nodes, during the forward phase of the RSVP-TE signaling, to ensure the required level of QoT. No wavelength conversion is assumed. The work in [7] evaluates the effect of using regenerators for both improving QoT and wavelength conversion, using the destination-designated strategy of the regeneration points [6]. A trade-off between the main performance metrics (blocking of LSP requests and average regeneration utilization) has been reported.

This paper proposes a new strategy, which aims at designating the regeneration points together with the WC points in a GMPLS-controlled WSON. The novelty of the proposed strategy is two-fold. First, the strategy is based on a fully distributed approach which is inline with the working principle of the RSVP-TE protocol. The regeneration points are designated in a distributed way during the backward phase of the RSVP-TE signaling session. For this reason, the strategy is named distributed-designation (DISTR). Second, the joint selection of regeneration and WC points is considered for the first time in a GMPLS dynamic network. In order to implement the DISTR strategy, a novel object vector is introduced in the Path message of the RSVP-TE protocol.

The aim of the work is to evaluate the benefits in terms of blocking, resource utilization, and fairness achievable by the proposed strategy. The performance of the novel strategy is compared with the best performing strategy for regeneration point designation from [6], i.e., the destination-designation (DEST) strategy described in the following section.

\section{Destination-Designation Strategy (DEST)}

During the RSVP-TE signaling session for establishing an LSP, the following standard objects are used in the Path message: the Label Set (LS), the Explicit Route Object (ERO), and the Record Route Object (RRO). Furthermore, two novel objects are used for regeneration designation according to [6]: Regeneration Availability Object (RAO) (in the Path message) and Regenerator Object (RO) (in the Resv message). LS elements indicate the wavelength availability. ERO contains 
the sequence of nodes forming the path. RRO contains the actual set of nodes comprising the full path. RAO contains the information of the available regenerators along the path, whereas RO contains the set of nodes designated to be regeneration points. Without loss of generality, it is assumed that QoT is guaranteed by limiting the maximum all-optical reach, referred to as maximum regeneration span [8].

In DEST, the designation of regeneration nodes is performed only at the destination based on the RAO object as indicated in Fig. 1 and cannot be overwritten by the intermediate nodes. Once the destination receives the RSVP-TE Path message, it is informed about the number of available $3 \mathrm{R}$ regenerators at each node. Based on such information, it proceeds to select the regeneration points by ensuring that the maximum regeneration span is met. If a $3 \mathrm{R}$ regenerator is not available in the node close to (but within) the maximum regeneration span, then the preceding node is examined. This is repeated until suitable regeneration points are found. The chosen regeneration points are indicated in the RO and put in the Resv message. Then, wavelength selection is performed.

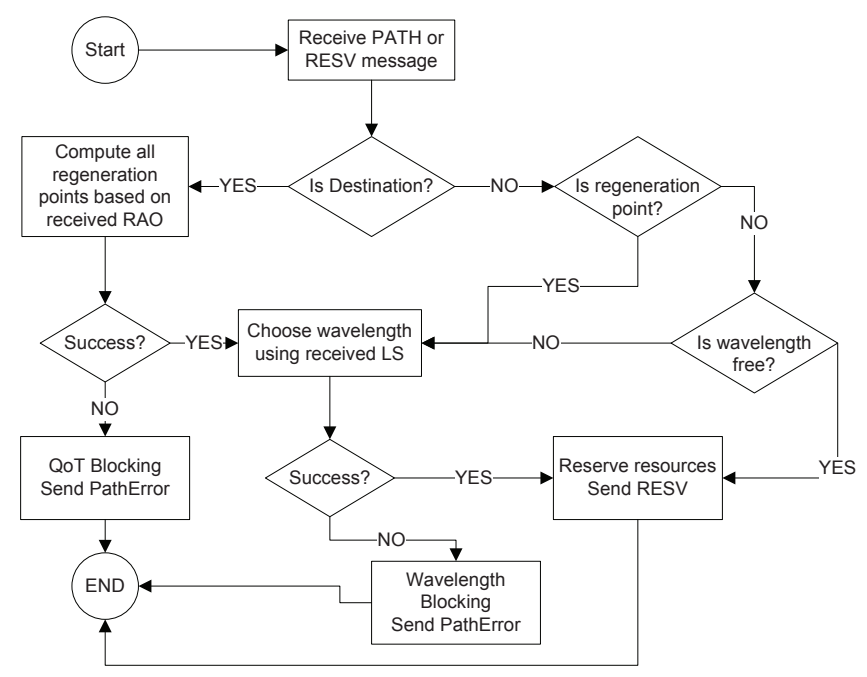

Fig. 1. Flow-chart for the DEST strategy.

During the backward reservation phase of RSVP-TE, each node that is a regeneration point (as indicated in the RO) reserves a regenerator and selects a new wavelength by applying a wavelength selection strategy on the stored $\mathrm{LS}^{1}$. Therefore, depending on the selected wavelength, the regenerator point may act also as a WC point. If the node is not a regeneration point and the previously selected wavelength is not available on the outgoing link, the node becomes a WC point and selects a new wavelength. In this case, a regenerator needs also to be reserved. In the case of unsuccessful reservation of wavelengths or regenerators, a PathError message is returned and the RSVP-TE instance is terminated.

Consider the example on Fig. 2. Assume that the maximum regeneration span is three hops and that regenerators are

\footnotetext{
${ }^{1}$ Note that under standard LSP establishment a new wavelength selection is performed only at wavelength conversion points, i.e., only if the wavelength is not continuous.
}

available at each node (as shown in the RAO object). The destination $E$ selects node $B$ as regeneration point. Depending on the wavelength selection strategy, a wavelength conversion may also be necessary. For instance, a first fit selection of the wavelength at the destination $\left(\lambda_{0}\right)$ forces node $C$ to be a WC point. Node $C$ applies the first fit strategy and selects $\lambda_{1}$. Such wavelength is unavailable on link $A-B$, thus node $B$ must selects a new wavelength (e.g., $\lambda_{2}$ ) for the last hop. Node $B$ acts as both a regeneration point and a WC point. Notice that the wavelength selection strategy affects the location and the number of $\mathrm{WC}$ points. For instance, if the destination node selects $\lambda_{3}$, wavelength conversion is not required and a single regenerator must be reserved at node $B$ for QoT purposes. Therefore, it is important that in the intermediate nodes wavelength selection and regenerator selection are performed jointly.

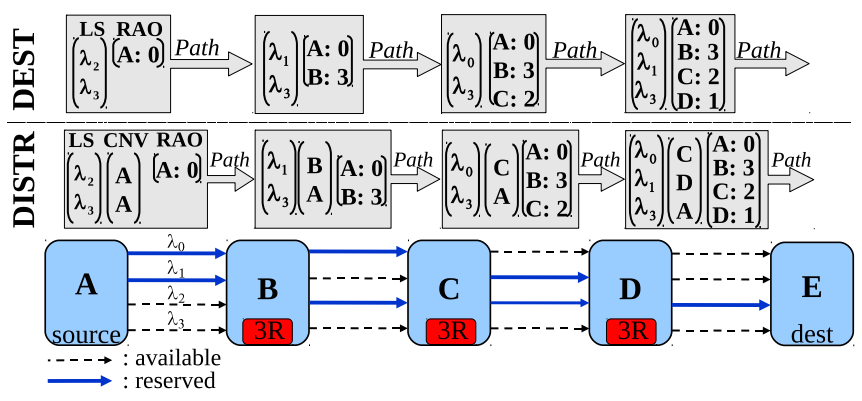

Fig. 2. Example of an LSP establishment with DEST and DISTR.

\section{Distributed-Designation Strategy (DISTR)}

Similar to the DEST strategy, the DISTR strategy requires the following objects in the Path message: LS, ERO, RRO, and RAO. In addition, a novel vector, proposed in this work, called Conversion Node Vector ( $C N V$ ) needs to be introduced. $\mathrm{CNV}$ includes an element for each wavelength contained in the LS, indicating the ID of the closest upstream node where wavelength conversion must take place for the corresponding wavelength. The RO object is used in the Resv message to indicate the designated upstream regeneration point.

\section{A. Example}

In order to explain the CNV usage, consider again the example displayed in Fig. 2. The maximum regeneration span is three hops and regenerators are available at each node. When selecting $\lambda_{3}$, the destination knows that the wavelength is available up to $A$ as indicated in the corresponding entry in the CNV and thus no wavelength conversion is required. Thus, the destination can designate the regeneration point(s) based on the regenerator availability indicated in the received RAO object and the maximum regeneration span, i.e., node $B$ is designated. Instead, if $\lambda_{0}$ (or $\lambda_{1}$ ) is selected, the destination knows that a conversion is required at node $C(D)$, i.e., before reaching the maximum regeneration span. Thus, the destination can proceed to designate jointly the next regeneration point and WC point, based on RAO and the maximum 
regeneration span. In particular, the destination can force node $C$ (node $D$ ) to act as both a WC and a regenerator point. The other main difference with the DEST strategy is that the intermediate node $C(D)$ determines the next regeneration and WC points, in addition to selecting a new wavelength. In this case, node $C(D)$ knows that $\lambda_{3}$ is available up to node $A$, which is still within the maximum regeneration span and no additional WC nor regeneration points are required. Therefore, in the considered example, one single regenerator is sufficient for any wavelength in the LS when the CNV vector is employed. Without the CNV (i.e. applying $D E S T$ ) two or even three regenerators might be required. The detailed description of the DISTR strategy is explained next.

\section{B. Description}

At the source node, each element of $\mathrm{CNV}$ is initialized with the source node ID for each wavelength included in the outgoing LS. For each wavelength included in the outgoing LS, the corresponding entry in the CNV is updated at each intermediate node, as follows. If wavelength conversion is locally required for a wavelength, the corresponding CNV entry is set to the node ID. Otherwise, the entry is left unchanged.

The sequence of operations performed by the DISTR strategy is summarized in Fig. 3. The strategy is implemented at the destination node (upon receiving a Path message) and at every intermediate node (upon receiving a Resv message). The destination node receiving a Path message jointly checks the regenerator availability in the RAO and the required conversion nodes indicated in the CNV. Based on such information, the destination can select at best the upstream regeneration point and/or WC point along with the wavelength to be used. The joint selection is carried out in the backward phase of RSVP-TE by performing three operations: LS pruning, wavelength selection, and upstream regeneration point designation. This procedure is performed during the backward signaling phase at the destination and at any intermediate node in which wavelength conversion and/or $3 \mathrm{R}$ regeneration is required.

The LS pruning is performed as follows ${ }^{2}$. First, the node which is the most distant node in upstream direction (based on the RRO), but still within the regeneration span is selected as a first regeneration reference point. Then, the destination node identifies the wavelengths in the LS which do not require any wavelength conversion and the wavelengths that require wavelength conversion at the regeneration reference point. The other wavelengths are pruned (i.e., removed from the LS). If all wavelengths are pruned, then a second run of processing is performed over the received LS by removing all wavelengths which require conversion in the downstream nodes from the considered regeneration reference point. If the pruned LS is still empty, then the LS is left unchanged.

Wavelength selection is performed on the pruned LS, according to any strategy (e.g., first-fit or random). The CNV

\footnotetext{
${ }^{2}$ From an implementation point of view, the pruning is performed over a copy of the LS and not the original LS
}



Fig. 3. Flow-chart for the DISTR strategy.

entry corresponding to the selected wavelength indicates the WC point. The regeneration point is decided as follows: (a) if the WC point is within the regeneration span, the WC point becomes also a regeneration point (i.e., wavelength conversion and regeneration are effectively combined together at the same node); (b) if the WC point is outside the regeneration span, the regeneration point is selected based on the regenerator availability indicated in the RAO and the considered regeneration span (as explained for the DEST strategy).

\section{Discussion}

In both presented strategies wavelength selection is performed at each regeneration point. However, in the DISTR strategy, the wavelength selection is performed only if the previously selected wavelength is not continuous up to the source node. Selecting a new wavelength at each regeneration point, even if not necessary, is more beneficial under the DISTR strategy. Indeed, by disseminating the information about the maximum transparent span of each wavelength unnecessary wavelength conversions are avoided especially in nodes close to regeneration points. An example is shown in Fig. 4. The maximum regeneration span is two hops. Thus, the destination selects node $C$ as a regeneration point. Assume that the destination selects wavelength $\lambda_{3}$. In absence of CNV, if node $C$ selects wavelength $\lambda_{3}$, a WC is required at node $B$, (i.e., two regenerators are required overall). Instead, by using the DISTR strategy based on CNV, node $C$ is able to see that $\lambda_{3}$ is not continuous up to the source, whereas $\lambda_{1}$ is. Thus, node $C$ selects wavelength $\lambda_{1}$ and only one regenerator is used.

\section{Simulation Scenario and Results}

The described strategies and their required protocol extensions have been implemented in the event driven simulator OPNET [9]. A Pan-European topology [10] with 28 nodes 


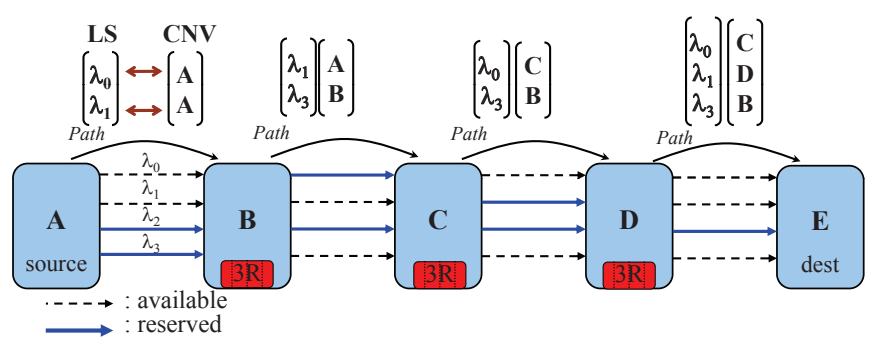

Fig. 4. Example of CNV usage with maximum regeneration span of 2 hops.

and 60 bidirectional links has been used, with 16 wavelengths per link and 16 regenerators per node. The regeneration span is assumed to be two hops. LSP requests are generated according to a Poisson process, whereas their duration is exponentially distributed with an average of $30 \mathrm{~min}$. The indicated traffic load is the total input traffic load in the network in Erlangs. The confidence interval bars at $95 \%$ confidence level are presented in the plots though not always distinguishable.

Four performance metrics have been evaluated: LSP blocking (Fig. 5), average number of regenerators used in the network (Fig. 6) average path length (Fig. 7), and achieved fairness level (Fig. 8). The last metric indicates how well the examined strategies serve connections with different path lengths. Two wavelength selection strategies are used: first-fit (FF) and random (RA).

Fig. 5(a) shows that the proposed DISTR strategy is able to lower the overall LSP blocking by up to one order of magnitude at low loads. The overall blocking probability is due to either the lack of wavelengths and/or regenerators for performing wavelength conversion (referred to as wavelength blocking Fig. 5(b)), or the lack of regenerators for ensuring QoT (referred to as QoT blocking Fig. 5(c)). In the considered scenario, since the QoT blocking is the predominant source of blocking (due to the chosen length of the regeneration span), the DISTR benefits are enhanced. QoT blocking is experienced in the backward phase of the RSVP-TE signaling, whereas the wavelength blocking is experienced in the forward phase. As a result, similarly to [11], RA outperforms FF when considering the backward blocking contribution and vice versa when considering the forward blocking contribution.

Under FF, wavelength utilization is "packed" towards the low indexed wavelengths. In DEST, this permits FF to experience a lower wavelength blocking compared to RA (see Fig. 5(b)). In DISTR, the pruning leads to a less "packed" wavelength utilization which brings the FF performance closer to RA performance. For the same reason, DISTR has a wavelength blocking higher than DEST, when using FF at medium and high loads. The much lower QoT blocking of FF DISTR compared to FF DEST however determines a lower overall blocking.

Fig. 6 shows the average number of regenerators used in the network. Depending on the load, the DISTR strategy reserves between $10 \%$ and $30 \%$ less regenerators with FF and between $10 \%$ and $17 \%$ less regenerators with RA with respect to the

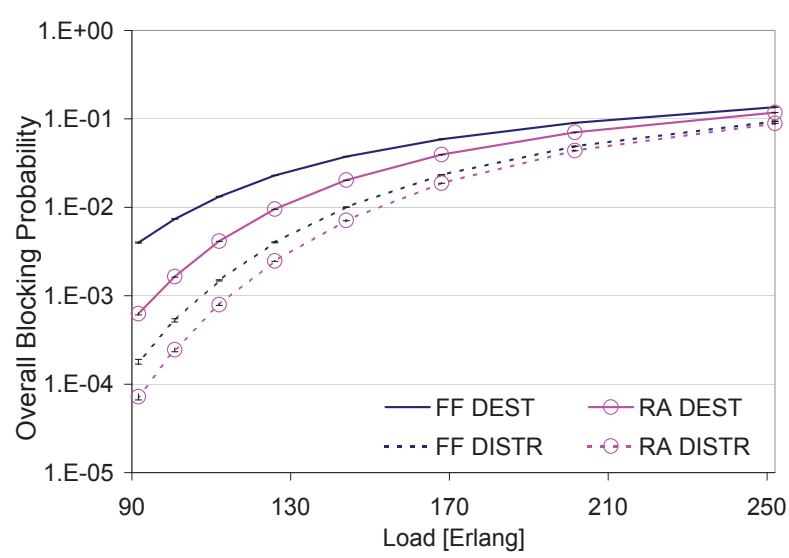

(a) Overall blocking probability vs. load.

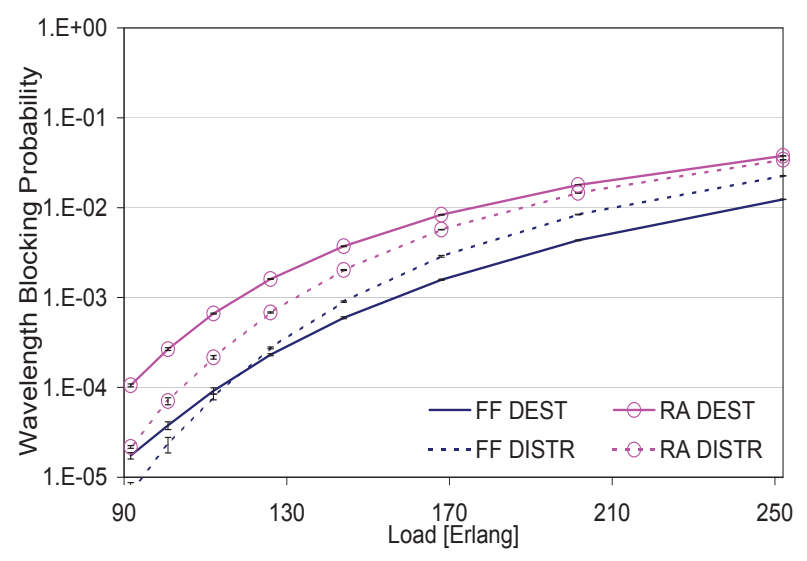

(b) Wavelength blocking probability vs. load.

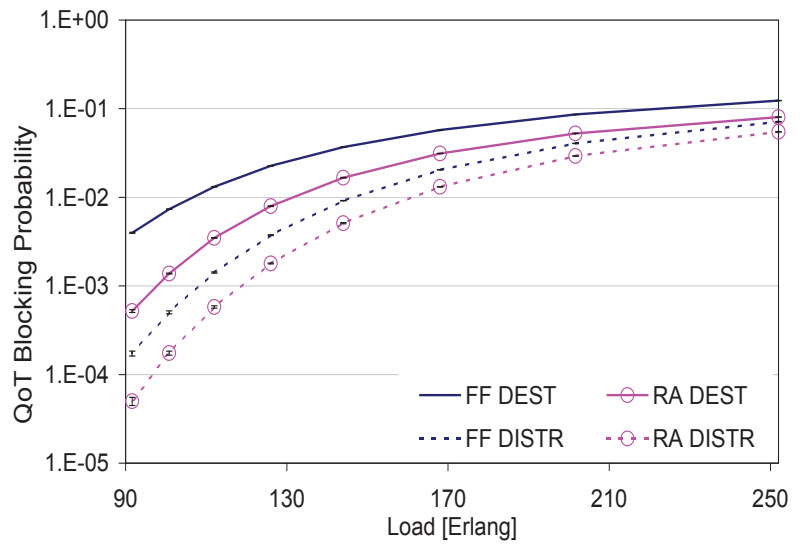

(c) QoT blocking probability vs. load.

Fig. 5. LSP Blocking probability vs. Load

DEST strategy, indicating the higher efficiency in saving these expensive and power-consuming resources.

Finally, Fig. 7 and Fig. 8 aim at quantifying the fairness of the strategies. Fig. 7 shows the average path length of the established LSPs as a function of the load, whereas Fig. 8 shows the distribution of the blocking probability as a function of the LSP path length with FF, for different load ranges. The average path length of the established LSPs is higher in the DISTR than in the DEST strategy at medium and high loads. 


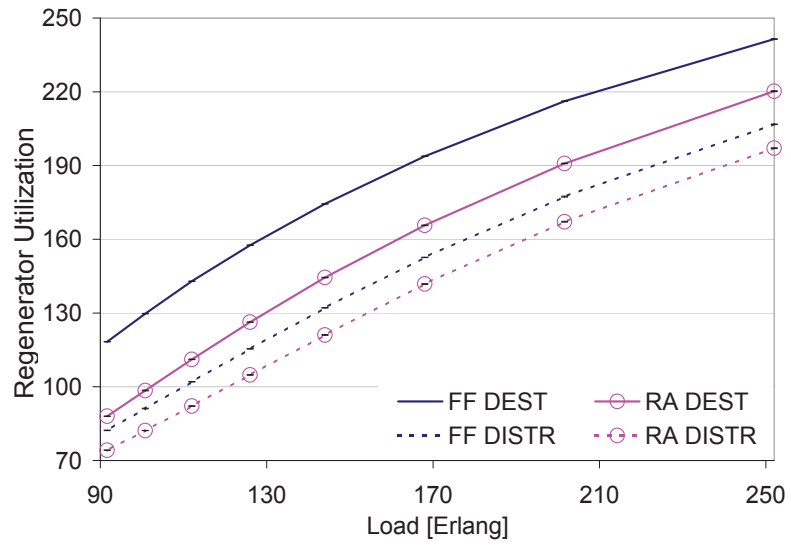

Fig. 6. Avg. regenerator utilization vs. load.

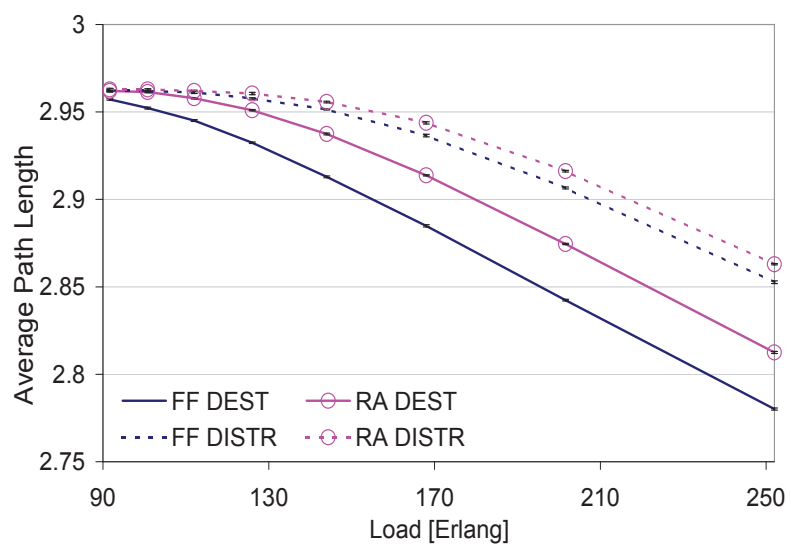

Fig. 7. Avg. path length for established LSPs vs. load.

This is due to the fact that although the blocking probability increases with the LSP length, the improvement of blocking probability achieved by the DISTR strategy is greater for long LSPs (i.e., longer paths have a better chance to be established). The degraded blocking performance of the shortest LSPs at medium and high loads is due to the increased wavelength blocking experienced by FF (see Fig. 5(b)). LSPs with path

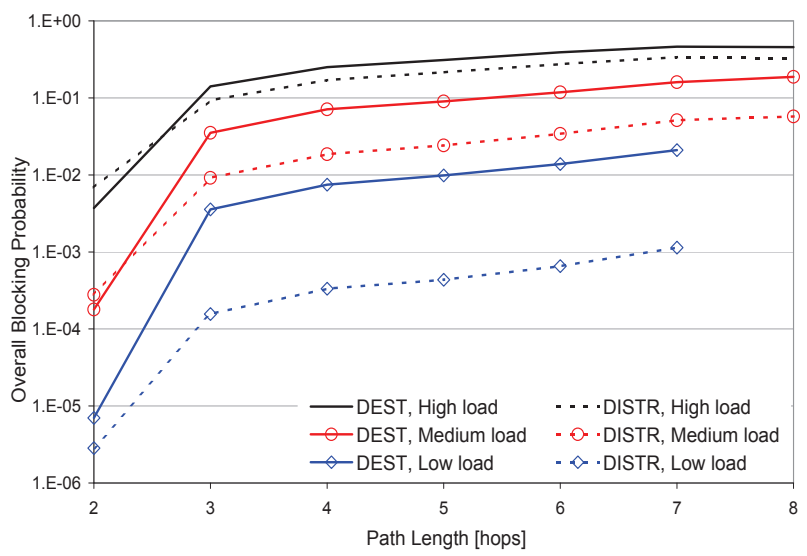

Fig. 8. Blocking probability vs. LSP length in number of hops, at different loads for FF wavelength assignment. length of two hops suffer only wavelength blocking and thus, DISTR strategy performs worse than the DEST. Under the given simulation setup, LSPs with path length of one hop did not experience any blocking.

\section{CONClusion}

A distributed strategy for joint selection of regeneration points and WC points has been proposed for RSVP-TE. The strategy aims at choosing regeneration points and WC points in such a way that they coincide as much as possible.

The proposed strategy has been shown to achieve three main advantages with respect to existing strategies: lower blocking, lower regenerator utilization, and higher fairness. The proposed DISTR strategy provides between $24 \%$ (for high loads) and $95 \%$ (for low loads) improvement in blocking; between $10 \%$ and $30 \%$ improvement in regenerator utilization; and improved fairness for long-distance connections, compared to the DEST strategy. Such advantages were achieved using random and first-fit wavelength selection and are expected to hold for any other wavelength selection. The proposed strategy is an intelligent method for optimally sharing the scarce and expensive regenerators between the impairment compensation and the wavelength conversion functionalities and fits well the distributed approach of GMPLS protocols.

Future studies can evaluate the impact of different $3 R$ allocation algorithms and extend the approach to a GMPLS control plane with Path Computation Elements.

\section{REFERENCES}

[1] S. Azodolmolky et al., "A survey on physical layer impairments aware routing and wavelength assignment algorithms in optical networks," Computer Networks, vol. 53, December 2009.

[2] M. Savasini et al., "Regenerator placement with guaranteed connectivity in optical networks," in Optical Network Design and Modeling, ser Lecture Notes in Computer Science. Springer Berlin / Heidelberg, 2007, vol. 4534, pp. 438-447.

[3] E. Mannie ed., "Generalized Multi-Protocol Label Switching (GMPLS) Architecture," RFC 3945, Oct. 2004.

[4] L. Berger ed., "Generalized multi-protocol label switching (GMPLS) signaling resource reservation protocol-traffic engineering (RSVP-TE) extensions," RFC 3473, 2003, IETF web site, Category: Standards Track.

[5] N. Andriolli et al., "Label preference schemes in GMPLS controlled networks," IEEE Comm. Letters, vol. 10, no. 12, December 2006.

[6] N. Sambo et al., "Accounting for shared regenerators in GMPLScontrolled translucent optical networks," J. Lightw. Technol., vol. 27, Oct. 2009.

[7] A. Manolova et al., "Wavelengths and Regenerators Sharing in GMPLScontrolled WSONs," in IEEE GLOBECOM, 2010.

[8] N. Sambo et al., "Accounting for PMD temporal correlation during lightpath set up in transparent optical networks," J. Lightw. Technol., vol. 28, Mar. 2010.

[9] OPNET technologies Inc., www.opnet.com.

[10] S. Ruepp et al., "Restoration in All-Optical GMPLS Networks with Limited Wavelength Conversion," Computer Networks Special Issue on Opportunities and Challenges in Optical Networks, vol. 52, no. 10, pp. 1951 - 1964, July 2008.

[11] A. Giorgetti et al., "Label preference schemes for lightpath provisioning and restoration in distributed GMPLS networks," J. Lightw. Technol., vol. 27, no. 6, pp. 688 - 697, March 2009. 